\title{
Non-targeted mass isotopolome analysis using stable isotope patterns to identify metabolic changes
}

\author{
Christian-Alexander Dudek ${ }^{1}$, Lisa Schlicker ${ }^{1}$ and Karsten Hiller ${ }^{1,2^{*}}$
}

1 Department of Bioinformatics and Biochemistry, Braunschweig Integrated Centre of Systems Biology (BRICS), Technische Universität Braunschweig, Rebenring 56, 38106 Braunschweig, Germany

2 Computational Biology of Infection Research, Helmholtz Centre for Infection Research, Inhoffenstraße 7, 38124 Braunschweig

\section{* karsten.hiller@tu-braunschweig.de}

\section{Abstract}

Gas chromatography coupled with mass spectrometry can provide an extensive overview of the metabolic state of a biological system. Analysis of raw mass spectrometry data requires powerful data processing software to generate interpretable results. Here we describe a data processing workflow to generate metabolite levels, mass isotopomer distribution, similarity and variability analysis of metabolites in a non-targeted manner, using stable isotope labeling. Using our data analysis software, no bioinformatic or programming background is needed to generate results from raw mass spectrometry data.

Keywords Gas chromatography, Mass spectrometry, GCMS, Data analysis, Metabolism, Mass isotopomer distribution, Stable isotope labeling, Non-targeted metabolomics.

\section{Introduction}

Gas chromatography (GC) or liquid chromatography (LC) coupled to mass spectrometry (MS) is widely used to measure metabolites in biological samples [1]. While classic metabolomics approaches only provide a static view of metabolite concentrations, metabolic flux analysis investigates dynamic metabolite conversion rates [2]. In this regard, flux balance analysis (FBA) relies on stoichiometric reaction models and predicts a solution space of feasible combinations of metabolic fluxes within the modeled network. This solution space 
can be reduced by thermodynamic constraints and by measured extracellular metabolic fluxes. On the other hand, ${ }^{13} \mathrm{C}$ metabolic flux analysis (MFA) provides exact metabolic fluxes for specific experimental conditions. It employs a combination of experimental data from stable-isotope labeling experiments and mathematical modeling of atom transitions. Both methods have in common that they rely on extensive prior knowledge of the metabolic reaction network and underlying stoichiometries [3] and often such a detailed information is not available a priori. Due to limitations of our biochemical knowledge, many metabolites have not yet been connected to a metabolic network or cannot even been identified after mass spectrometry. To overcome some of these limitations, we established a non-targeted mass isotopolome analysis, which allows for the identification of metabolic fluxes without a priori knowledge of the underlying metabolic network [4]-[7].

The initial part of such a study is a stable-isotope labeling experiment. For this purpose, a ${ }^{13} \mathrm{C}$, ${ }^{15} \mathrm{~N}$ or any other stable-isotope tracer is applied to the biological system and tracer derived isotopes are incorporated into downstream metabolites. After metabolite extraction and massspectrometry driven measurement, all detectable known and unknown isotopically enriched metabolites can be identified with algorithms such as non-targeted tracer fate detection (NTFD), MetExtract or 13CXCMS [8]-[11]. In a next step, mass isotopomer distributions (MIDs) for all detected and thus labeled metabolites are determined based solely on the massspectrometric data of the non-labeled counterparts as no structural information is available at this point [7], [8], [12]. All these MIDs are determined by metabolic fluxes through the underlying biochemical reaction network and can be applied to reveal both, the network structure and metabolic flux changes [7], [9].

In the following we will describe in detail two options for a non-targeted mass isotopolome analysis (Fig 1).

\section{Materials}

The following software packages are required to perform the described non-targeted analysis of GC-MS data recorded in the context of a stable-isotope labeling experiment. 


\subsection{MetaboliteDetector}

MetaboliteDetector is a software designed for the semi-automatic analysis of GC-MS raw data. The software provides a graphical user interface and can be applied for targeted and non-targeted approaches. For that, MetaboliteDetector implements an extensive set of different GC-MS data analysis algorithms, including retention time correction, ionchromatographic deconvolution, metabolite detection, metabolite identification, metabolite quantification and first statistical analyses. Moreover, this program can be applied to determine MIDs for target compounds and correct these for natural occurring isotopes. Finally, a wide variety of data visualization options is available, like single- and total ion chromatograms, box plots and MID bar plots [13].

MetaboliteDetector is freely available for Linux operating systems at http://metabolitedetector.tu-bs.de.

\subsection{Mass Isotopolome Analyzer}

The Mass Isotopolome Analyzer (MIA) is a tool to visualize labeled metabolites in a MID similarity-based network. Additionally, a variability analysis of the different experimental conditions can reveal metabolic flux changes. MIA first detects all isotopically enriched metabolites present in a GC-MS data set [8] and then contextualizes MIDs of labeled compounds in a network visualization. A graph is created which connects metabolites with similar MID patterns based on dynamic programming and distance calculation. Different cutoffs for distance measurement and inter-experiment MID variability can be applied to analyze the datasets [7]. If a reference compound library is supplied, detected metabolites can be identified.

MIA is freely available for Windows and Linux operating systems at https://mia.bioinfo.nat.tu-bs.de

\subsection{Optional: Metabolite spectra reference libraries}

While the approaches described below are all of a non-targeted manner, the biological interpretation needs an incorporation of the non-targeted data into a known metabolic context. Therefore, identification of as many metabolites as possible is of advantage. 
Both, MetaboliteDetector and MIA can import and export reference libraries in MSL format and as such the Golm Metabolome Database (GMD) can be applied for compound identification. It is freely available [14] and currently contains 26,590 spectra of 3,568 derivatized analytes of 2,222 different metabolites and additionally 3,488 spectra of purchased reference substances from GC-MS measurements. See Note 1 on how to convert a library in MSL format into MetaboliteDetector format.

\section{Methods}

\subsection{Experimental prerequisites}

Any non-targeted detection of isotopic labeled metabolites starts with an experimental setup applying a stable isotope labeled metabolite as a tracer. In general, any stable isotope, for example carbon $\left({ }^{13} \mathrm{C}\right)$ or nitrogen $\left({ }^{15} \mathrm{~N}\right)$, can be employed. For every condition, two experiments are needed, one using the isotopic tracer and the other using the same substrate, but without enrichment. To get the best results, both experiments should be performed simultaneously under identical experimental conditions. It is also advised to perform biological replicates to validate the results of mass isotopomer calculations [7], [8]. As the retention time of analytes varies between instruments and different GC-MS columns, it is recommended to measure a mix of $n$-alkanes (usually $1 \mu \mathrm{L}$ of a mixture of $\mathrm{C}_{10}$ to $\mathrm{C}_{40}$, splitless injection) along with the samples. This enables the calculation of retention indices for improved metabolite identification in addition to spectrum similarity alone.

The detailed experimental procedure for cultivation and metabolite extraction highly depends on the metabolites and organism of interest. Different experimental protocols are described elsewhere [9], [15]-[20]. After GCMS measurement, the raw-data need to be exported into the common netCDF format, which can usually be done with the instrument software.

\subsection{Data import}

The protocol starts with the raw data in netCDF format. If not otherwise stated, the default settings are a good starting point. First, all netCDF files are imported with MetaboliteDetector's netCDF import function (Fig 2). Depending on the computational 
power, data import can take some time. During the import process, files with extension .bin and .idx are created in the same directory as the netCDF files, if no other output directory was selected.

\subsection{RI calibration and deconvolution}

As mentioned before, the retention index calibration is a highly recommended step because the retention time of metabolites can shift slightly, especially when measuring many samples. MetaboliteDetector provides the RI-Calibration Wizard, which guides through the calculation of retention indices and ion-chromatographic deconvolution. Measured $n$-alkanes need to be selected from the reference spectra library (Fig 3 left) and after detection of the alkanes, the calibration table lists the detected compounds, detected retention time and the corresponding retention index provided with the library (Fig 3 right). At this point it is crucial to validate that the peak-alkane assignment is correct and needs to be corrected otherwise. In the next step, deconvolution settings for compound detection need to be defined and should be applied according to your experimental setup (see Note 2).

After deconvolution and retention index calculation, the data is ready to be further analyzed in MetaboliteDetector or MIA. Since this step is crucial for all downstream analyses, it is highly recommended to verify the quality of the results of the deconvolution and RI calibration process (see Note 4).

\subsection{Similarity analysis}

For the interpretation of non-targeted metabolomics data, it is of advantage to first set (known) parts of the obtained results into a biological context and map unidentified labeled metabolites based on MID similarity to this established frame. In this regard, MIA provides networks based on MID similarities and can highlight variability of MIDs and thus underlying metabolic fluxes between different conditions (e.g. control and treatment) within this network.

A MIA analysis starts with the import of labeled and unlabeled chromatograms preprocessed by MetaboliteDetector (see previous paragraph) for every experiment (Fig 4A). Next, some settings need to be defined: For the compound identification settings, the RI Tolerance 
setting is the maximum retention index tolerance for library matching (smaller values require stricter matching), the Compound identification cutoff score is the minimum score needed for compound identification ( 1 - perfect match, 0 - no match) and Show top $n$ hits is the number of library matches for potential compound identification and name assignment. In the label detection section, Maximum fragment deviation is the maximum deviation of the summed MIs from 1.0. The summed fractions of all mass isotopomers should be 1.0 because each MI is typically given in percental values, Minimum number of labeled fragments is the minimum number of fragments per compound which need to be detected as labeled in order to get recognized as labeled compound, Required amount of isotopic enrichment is the minimum amount of labeling needed, Minimum $R^{\wedge} 2$ is the minimum coefficient of determination $\left(R^{2}\right)$ from MID determination, Minimum MO abd. is the minimum abundance of the unlabeled mass isotopomer (M0) needed and Ignore compounds with $M \_n \mid$ with $n>\ldots$ is the number of mass isotopomers from which a compound gets excluded. Additionally the Gap penalty for the Needlman-Wunsch-Scoring for MID alignment and the initial Distance cutoff for graph edges can be defined. It is highly advisable to select the same settings for every experiment to achieve better comparability across experiments. The settings for every experiment can be saved in XML format for future runs of the program (Fig 4B). After starting the isotope detection and MID profiling, every labeled metabolite will be represented by a node depicting the MID in the main window of MIA. In addition, all labeled metabolites will be listed in the left side of the window. Up till now the complete procedure is nontargeted and does not require any structural information on the detected metabolites. However, a compound library for identification can be applied at this stage to bring parts of the data into a biochemical context.

With the experiments and identifications in place, various options for graph layout, distance calculation (Fig 4C) and normalization (Fig 4D) can be applied. In most cases the default values (Canberra distance and normalization by sum) should be sufficient. The layout engine defines the way the nodes will be arranged in the network and should be selected based on the amount of nodes. While the "dot" layout is a general purpose engine, "circo" of "twopi" are suitable for fewer connected nodes and small clusters (Fig 4E). The distance cutoff sets the minimum similarity that two nodes need to have to get connected (Fig 4F). While increasing this cutoff, the nodes will start to rearrange and connect to each other based on 
their MID similarities. The edge color indicates the corresponding experiment. Unconnected metabolites can be hidden from the visualization through the options menu. Connected metabolites are similar based on their MIDs and in many cases be metabolically related.

\subsection{Variability analysis}

Metabolism is a highly dynamic but regulated system and can switch to different states depending on the environmental conditions. In many cases, changes in metabolic fluxes are not directly linked to changes of the metabolome or metabolite levels and are thus hidden in metabolomics analyses. However, stable isotope labeling has the power to reveal changes in underlying fluxes and for this purpose, MIA provides an MID variability analysis to detect flux changes between experiments. Using the previously created graph of metabolites with similar MIDs, the variation cutoff slider can be increased slowly (Fig 4G). While increasing the cutoff, nodes will start to disconnect, if the variability between experiments does not match the given cutoff. Additionally, if Hide others (Fig 4H) is enabled, the nodes will not only disconnect, but also disappear from the graph. All remaining connected nodes will have at least the given MID variation between experiments as defined by the cutoff slider.

\subsection{Library export}

All detected MIDs can be exported in CSV format for further processing with other programs. Additionally, MIA can generate and export a MetaboliteDetector library containing all detected labeled metabolites. This library can then be opened in MetaboliteDetector to perform additional sensitive profiling such as quantification or a more sensitive MID determination in a targeted manner (Section 3.7 and Section 3.8).

\subsection{Targeted search for quantification}

While MIA is a powerful tool to detect labeling in metabolites and create networks based on MID similarity, it lacks features for metabolite quantification and statistical analysis. Furthermore, MIAs sensitivity for MID determination is lower due to the non-targeted approach. To overcome these shortcomings, it is possible to apply MetaboliteDetector and perform a "non-targeted targeted” search for all metabolites detected by MIA. Because MIA provides exact mass spectra and retention times for all revealed known and unknown labeled 
compounds, these metabolites can be further profiled in MetaboliteDector even if their structure is not known.

To obtain semi-quantitative metabolite levels for MIA compounds with MetaboliteDetector, the metabolite library exported by MIA can be used (Tools - Batch quantification...). The batch quantification wizard will guide through the settings (Fig 5). First, the files need to be selected as replicate groups (See Note 3). Next, as analysis type, Targeted Analysis needs to be selected, because only the previously detected metabolites from MIA are of interest for this analysis. The last panel of the quantification wizard contains different settings:

- Ref. library refers, in this case, to the library of labeled compound spectra supplied by MIA.

- The retention index difference $(\Delta R I)$ is the maximum difference of retention indices of two compounds to get matched. A small value like 5.0 should be appropriate, but highly depends on the calibration quality (See Note 4).

- The identification score (Req. Score) defines the strictness of mapping to compounds together. Higher values require higher similarities in terms of spectrum and retention time to be matched. To avoid matching of compound with different structures, this value should be set to a higher value (e.g. 0.9).

After finalization of calculation, a new tab (Batch Quantification) appears in the bottom of the window and contains the quantification results. Each row represents one of the metabolites previously detected with MIA and is named accordingly. The data can be normalized by the summed sample signal $\left(\frac{1}{\sum \square}\right)$ or by the peak integral of a selected metabolite, in most cases an internal standard $\left(\frac{1}{A}\right)$. The statistics sub-tab presents the mean (and normalized if selected previously) intensities of the replicates. Additionally, the $p$-value (ANOVA) is calculated as a measurement for the statistical difference between the groups. 
Selected rows of the statistics sub-tab can also be displayed as a box plot. From the quantification sub-tab and the statistics sub-tab, the data can be exported in CSV format.

\subsection{Targeted search for MIDs}

Another option is to determine MIDs for all MIA compounds. This can be useful if MIDs could not be detected for some conditions within MIA or if additional chromatograms should be processed. However, for classical MID determination the sum formula needs to be supplied for each target compound to correct for natural occurring isotopes. Since this information is not available due to the non-targeted character of the MIA analysis, MetaboliteDetector implements an algorithm to correct MIDs for natural isotopes just based on the unlabeled compound spectrum that was provided at the beginning of the MIA analysis [12].

To perform such an analysis, the library exported by MIA needs to be loaded first (Tools Settings... - Identification - Compound Lib). Then, the MID wizard (Tools - MID Wizard...) has to be opened, which will guide through all required settings (Fig 6). In the analysis \& method panel, Corrected using library needs to be selected, because sum formulas for compounds are not available in this case (see above). Next, all files from the tracer experiment need to be selected as replicate groups. The last section of the MID wizard contains the same settings as described in Section 3.7. Here it is important to select the previously created library containing the unlabeled spectra from MIA.

The MIDs tab will appear in the bottom of the window when the analysis is finished. In the MIDs sub-tab, selected metabolite MIDs can be visualized as bar-plots. Additionally, the confidence interval (CI) and $R^{2}$ of the MID determination for every file is shown for quality control. The MID results should be revised carefully, as the non-targeted approach may return wrong ions, see Note 5 . The statistics sub-tab shows the means and standard error of the replicates. The MIDs can be exported in CSV format from the MID sub-tab. The data from both tabs will be exported in one file with different formats. This should be kept in mind for further automatic data processing. 


\subsection{Biological interpretation}

In the previous sections, different data have been created, that represent different aspects of the metabolic state of the analyzed sample. On one hand, the metabolite levels represent the total abundances of all (non-targeted) detected metabolites. On the other hand, the MID data represent the flux changes between metabolites, which can either correlate with the metabolite levels, or can even reveal flux changes, which are not visible in the metabolome dataset alone. Additionally, MIA provides tools to create metabolic connections based on MID similarity and variability analysis between different experiments. MID similarity based networks can reveal metabolite connections between known and unknown metabolites. The variability analysis can highlight connections where metabolic fluxes change between experiments or on the other side, show parts of a metabolic network which do not change between different experiments. Bringing these data into known metabolic context can reveal new metabolic interconnections and thus provide a better understanding of the observed phenotype.

\section{Notes}

\section{MSL library import and manipulation}

The MSL file format is a text format for mass spectrometric data, which can be imported into MetaboliteDetector using the library import function. The selected library in MSL will get scanned and the number of metabolites in the file will be displayed prior conversion. After the import, a library file in LBR format will be created. After conversion the new library can be automatically loaded or opened later through the settings dialog.

Libraries in MetaboliteDetector format can be opened within MetaboliteDetector using the Library Editor. All library compound data, like spectrum, meta data, ions for quantification and MID determination can be reviewed and revised if needed (Fig 7).

\section{Deconvolution settings}

Ion-chromatographic deconvolution is essential for the detection and separation of mass spectra of co-eluting metabolites. Therefore, the adjustment of the deconvolution settings is 
of high importance. The specific value depends on the GCMS instrument used for measurement. Below is a brief description of the parameters.

- Peak threshold must be exceeded by the 1st derivative of the peak function. Peaks below the threshold will be discarded. A lower value increases the sensitivity.

- Minimum peak height above the baseline. Peaks below this value will be discarded. A lower value increases the sensitivity.

- Bins/scan: The number of bins created for every scan before deconvolution. A lower value decreases the ability to separate co-eluting compounds, a too high value falsely splits compounds. Normally a value of 10 should be optimal. For detailed description see Stein et al. 1999.

- Deconvolution width is the maximum number of scans by which a chromatographic peak can differ to still be considered to belong to the same mass spectrum. If the value is too low, co-eluting compounds will not be separated, if the value is too high, peaks belonging to one compound will be falsely split in two or more.

- Required intensity (\% of base peak) for peak detection. Peaks assigned to a compound below this value will be discarded. Should be 0 in most cases.

- Required number of peaks for a metabolite to get detected. Deconvoluted metabolite spectra with less ions will not be displayed as detected metabolite.

Table 1 lists different settings for some GCMS instruments as reference. Additional fine tuning of the parameters is needed depending of the measured samples. However, the settings are fairly robust when using the same instrument and sample type.

\section{Replicate file grouping}

Defining replicate groups in MetabolieDetector can be tedious especially for large datasets. MetaboliteDetector provides a smart replicate group function (lightbulb symbol in Batch quantifaction or MID wizard) which is based on regular expressions. If replicate file names were consecutively numbered, the smart selection function automatically defines replicate groups for further processing and subsequent statistical analysis. For example, if the replicates were named like “expA_1”, “expA_2”, ...and “expB_1”, “expB_2”, the regular 
expression “( . * $)$ ”, will group all corresponding files in group “expA” and “expB” respectively.

\subsection{Spectrum quality verification}

The deconvolution and retention index calibration have a high impact on quality of the extracted chromatograms and matching of metabolites across samples. Therefore, it is important to revise the results of Section 3.3 before proceeding. The first step is to evaluate the quality of the total ion chromatogram. Overloaded peaks that exceed the detector limit have a negative impact on the retention time of following compounds and will not be deconvoluted correctly. If too many peaks are overloaded, it may be advisable to repeat the measurement with diluted extracts or a higher split.

Retention indices of the same metabolite should not differ too much between different samples. In some cases the $\frac{1}{\Sigma a} \mathrm{RI}$ can be adjusted to allow more RI deviation, but a small RI difference is preferable. If the retention indices differ between samples, the retention index calibration should be repeated. Especially the automatic detection of the alkanes should be revised carefully.

The single ion chromatogram in MetaboliteDetector can be used to quality control the results of the deconvolution. The different fragments (ions) of a detected metabolite should show the characteristic pattern with decreasing intensities for the mass isotopomers (Fig 8), which depend on the natural abundance of isotopes (mainly ${ }^{13} \mathrm{C}$ ) and the number of atoms. Peaks which do differ from this decreasing pattern may originate from overlapping signals which have not been correctly deconvoluted. While these artifacts cannot be excluded completely, the deconvolution settings should be adjusted to minimize those errors, especially for identified metabolites and metabolites of interest.

\subsection{MID quality verification}

The non-targeted approach will calculate MIDs for every ion which has been previously detected as labeled. This may lead to many different ions for one metabolite with different MID results. Due to fragmentation during the ionization process, the number of detected isotopomers can vary between the different ions. Here it is important to evaluate if the 
number of mass isotopomers matches with the detected metabolite. For example, with ${ }^{13} \mathrm{C}$ tracer, a metabolite with six detected mass isotopomers, which was been identified as lactic acid (maximum 3 labeled carbon atoms) does not match. Here it is possible, that only one particular ion was detected with the wrong amount of mass isotopomers. In this case it would be possible to exclude that ion from the generated library using MetaboliteDetectors library editor. If all ions are consistent and do not match the expected number of mass isotopomers, the identification may be wrong.

Another critical point is to evaluate the MID pattern based on knowledge of biochemical pathways. Using a specifically labeled tracer, one would expect a specific pattern for metabolites downstream of the tracer. These expectations should be evaluated with corresponding metabolites which have been identified. 


\section{Figures}

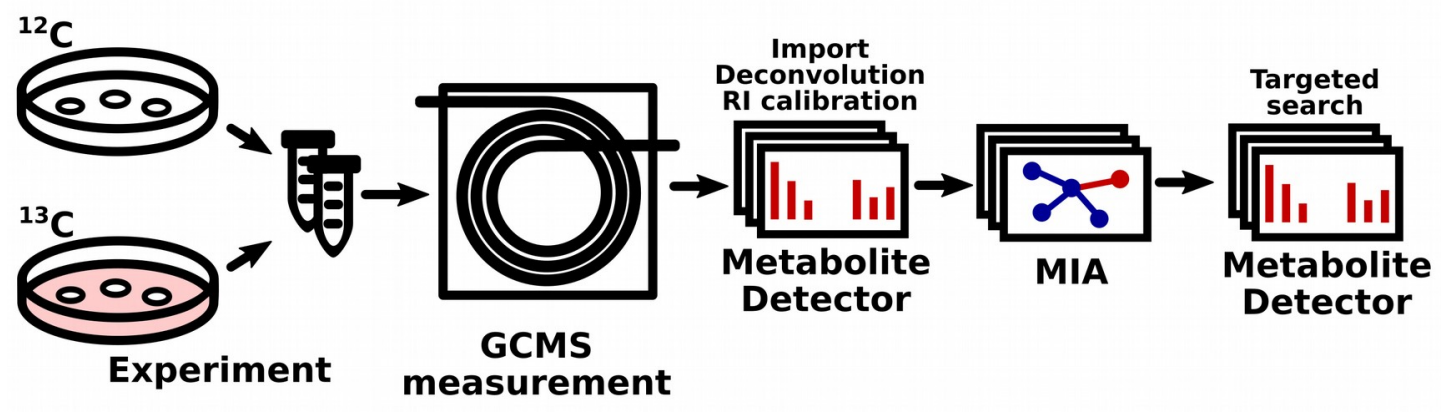

Figure 1: The presented workflow of a non-targeted experiment and data analysis. The workflow starts with the cell culture experiment and the preparation of metabolite extracts followed by gas chromatography and mass spectrometry (GCMS) measurement. The raw data in netCDF format needs to be imported, calibrated and devonvoluted in MetaboliteDetector. The similarity and variability analysis to create a network based on MID similarity is performed with MIA. For the subsequent targeted data analysis, MetaboliteDetector can be used to obtain more sensitive MID determination and quantification data. 


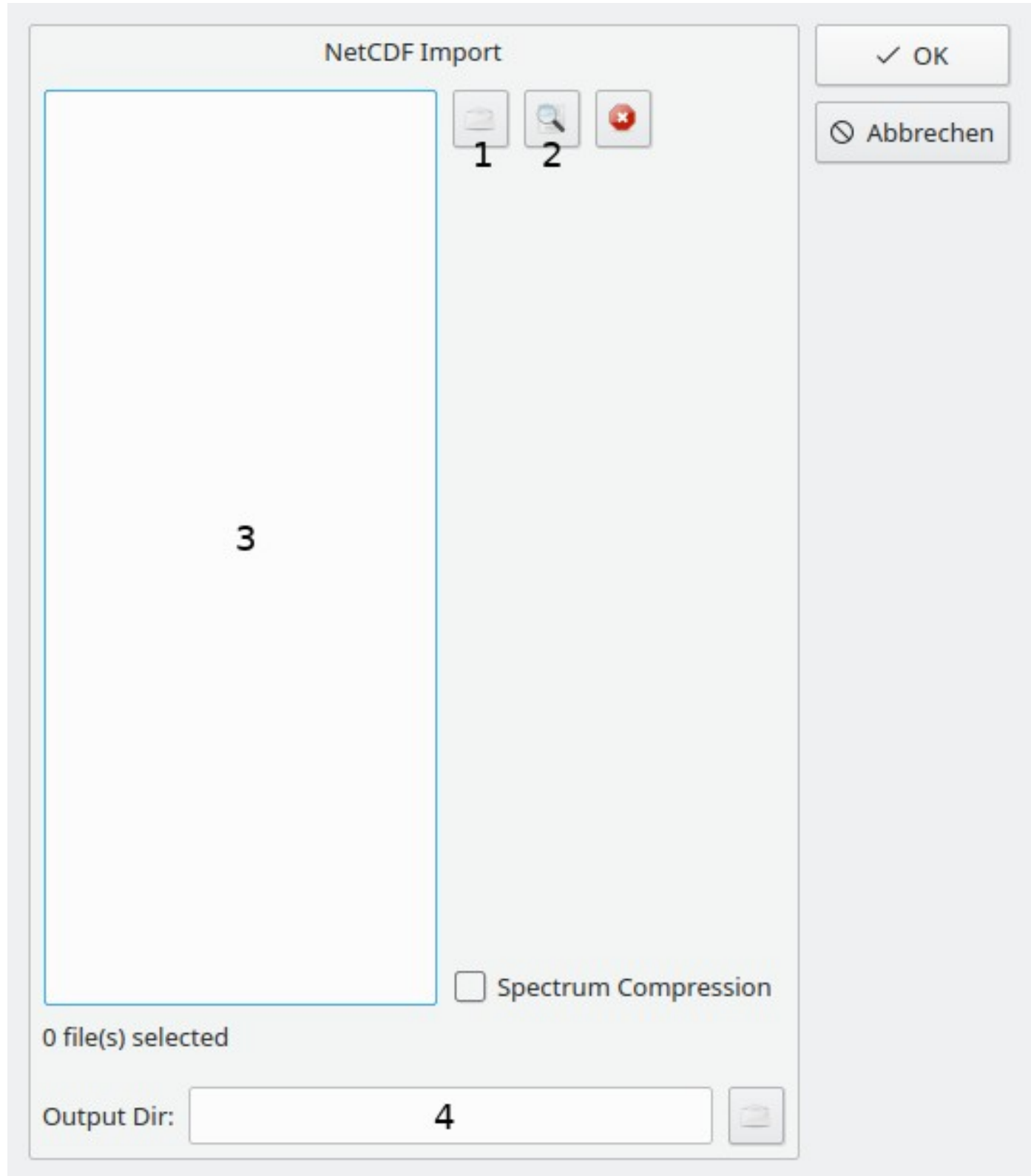

Figure 2: The netCDF import dialog. Files for import can be selected directly (1) or searched for recursively in a folder (2). The selected files will appear on the left (3). Optionally, a different output directory can be selected (4). 

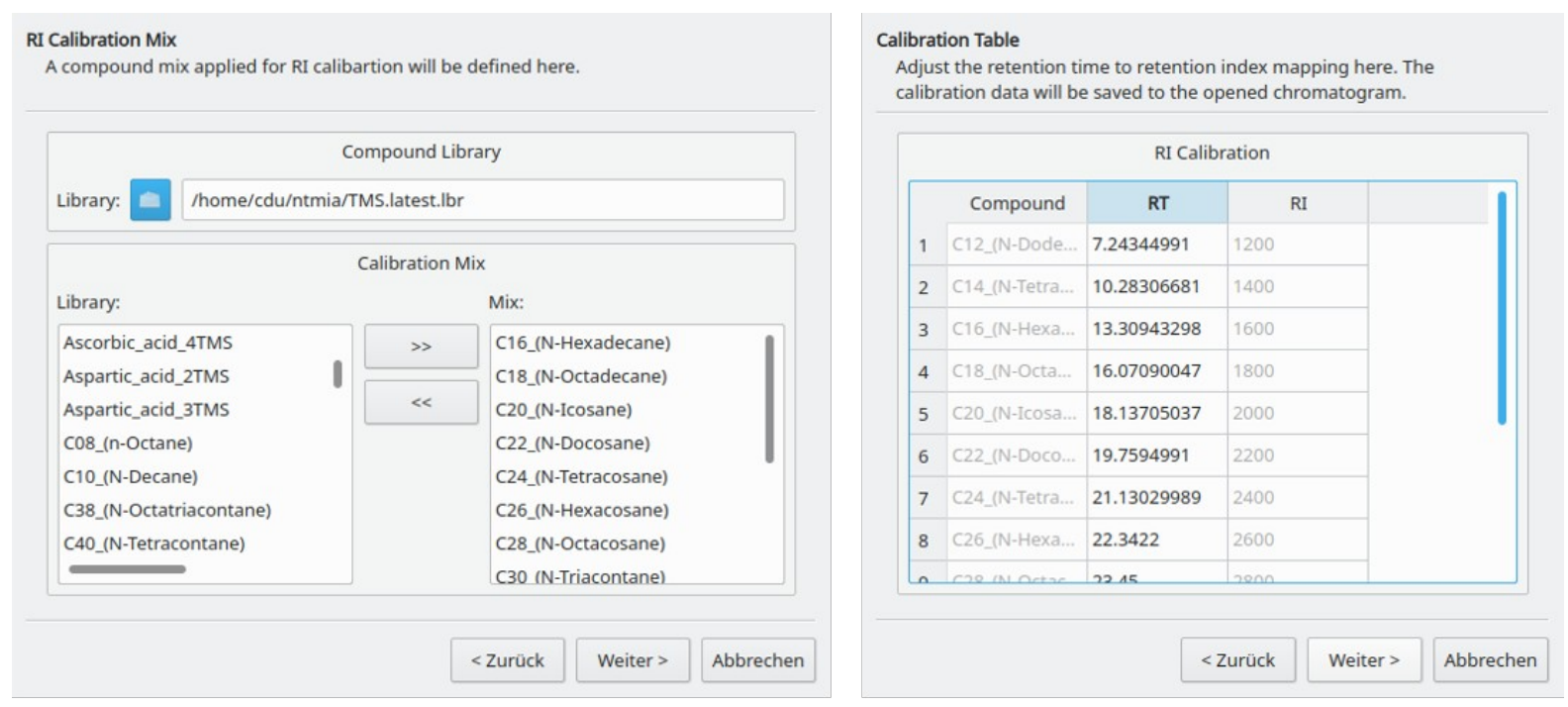

Figure 3: RI-Calibration Wizard configuration. Left: Selection of alkanes from the library. Right: Calibration table with alkanes, detected retention times and corresponding retention index from the library. 


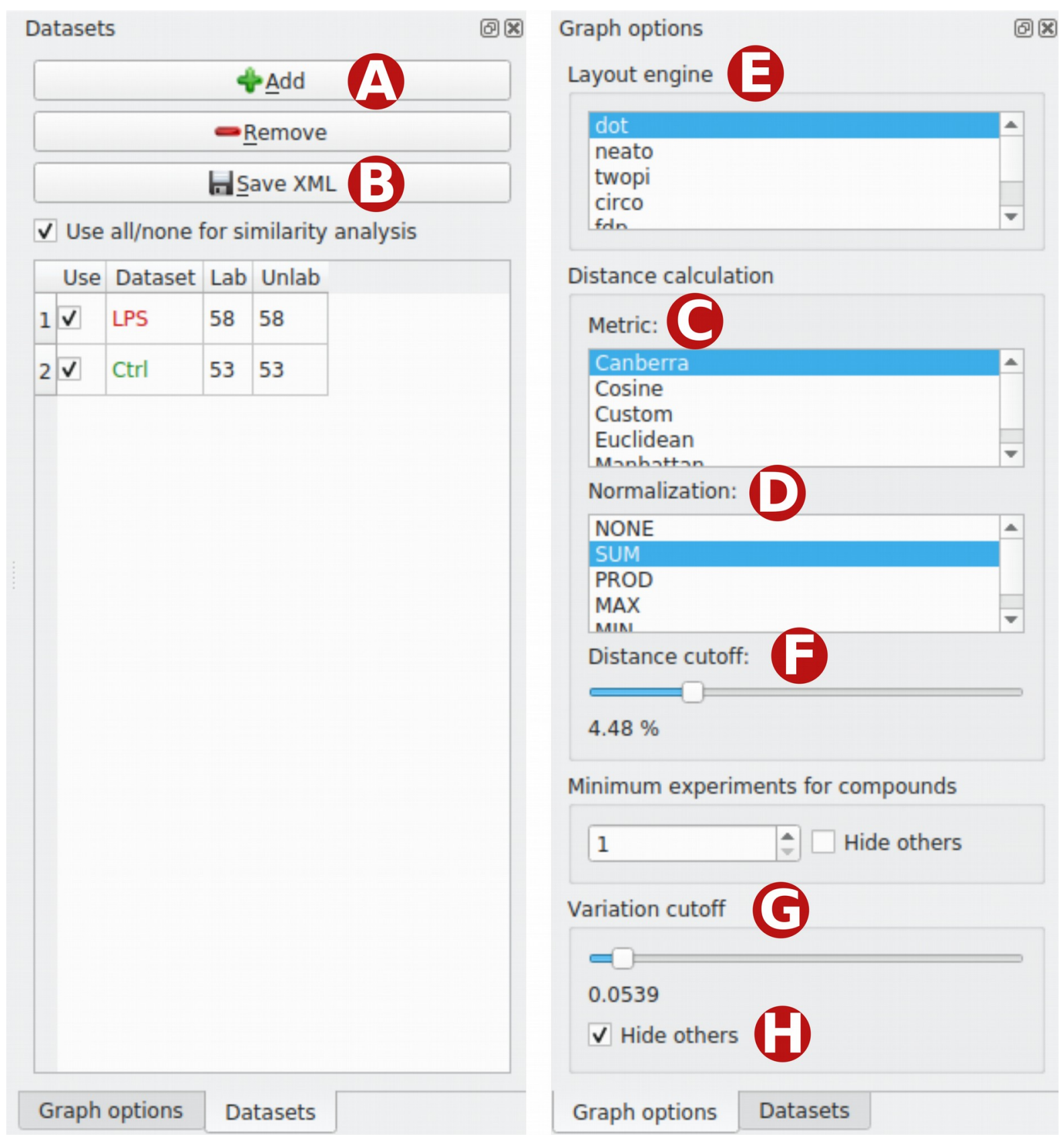

Figure 4: MIA option tabs. Left: In the datasets tab new experiments can be added (A), removed or saved (B). Additionally, all experiments are listed with the corresponding color and number of labeled and unlabeled metabolites. Right: In the graph options the layout engine (E), distance metric (C) and normalization (D) can be selected. Using the distance cutoff slider (F) the minimum MID similarity distance for node connection can be adjusted. The variability slider $(G)$ can be used to only show connections for nodes with MID variation between experiments. If Hide others is checked $(\mathrm{H})$, all other nodes will not be shown. 


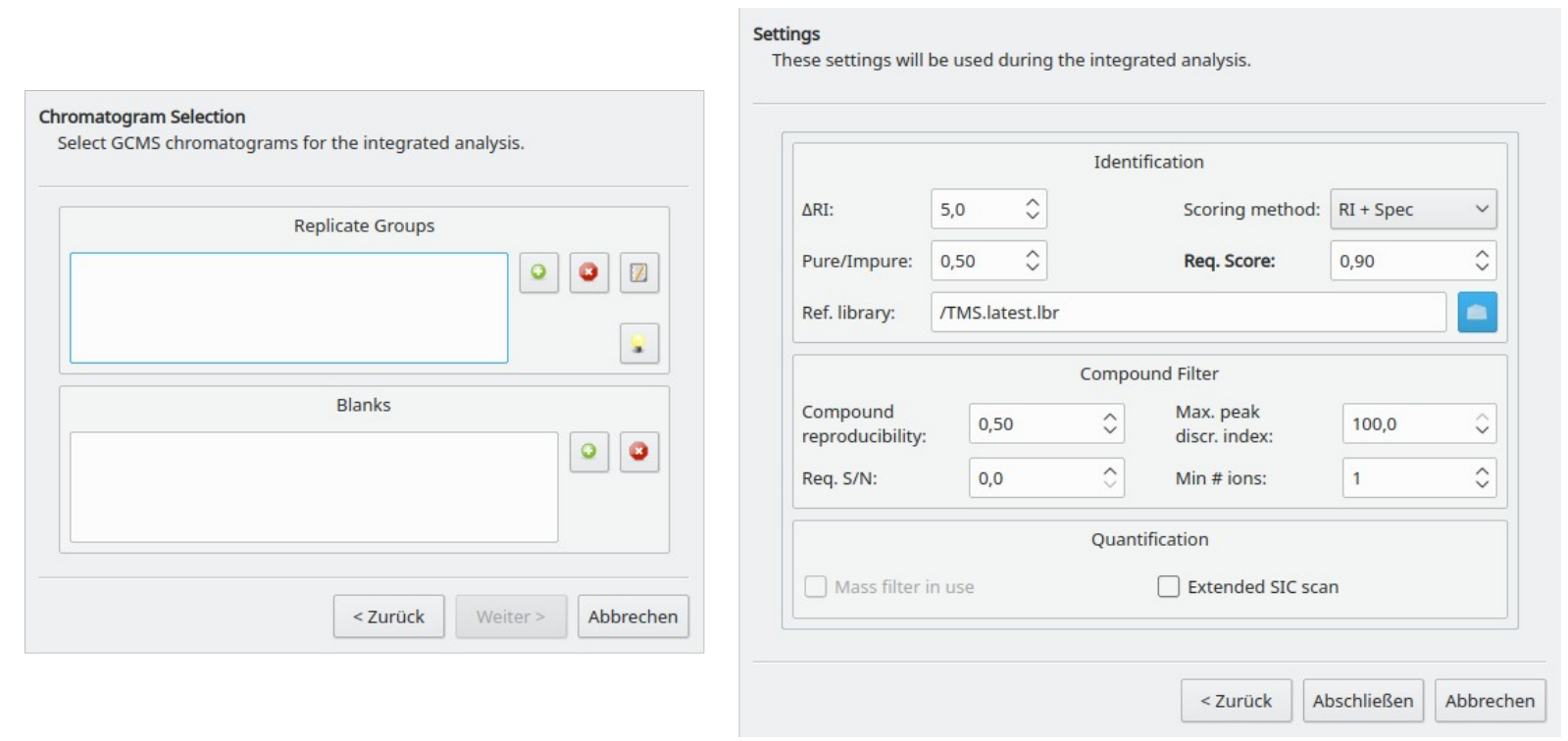

Figure 5: Batch quantification wizard. The files for replicates (left) can either be select manually, using the plus button, or alternatively using the lightbulb button (see Note 3) for regular expression based replicate selection. The settings window (right) contains the settings for metabolite identification and additional compound filtering. 

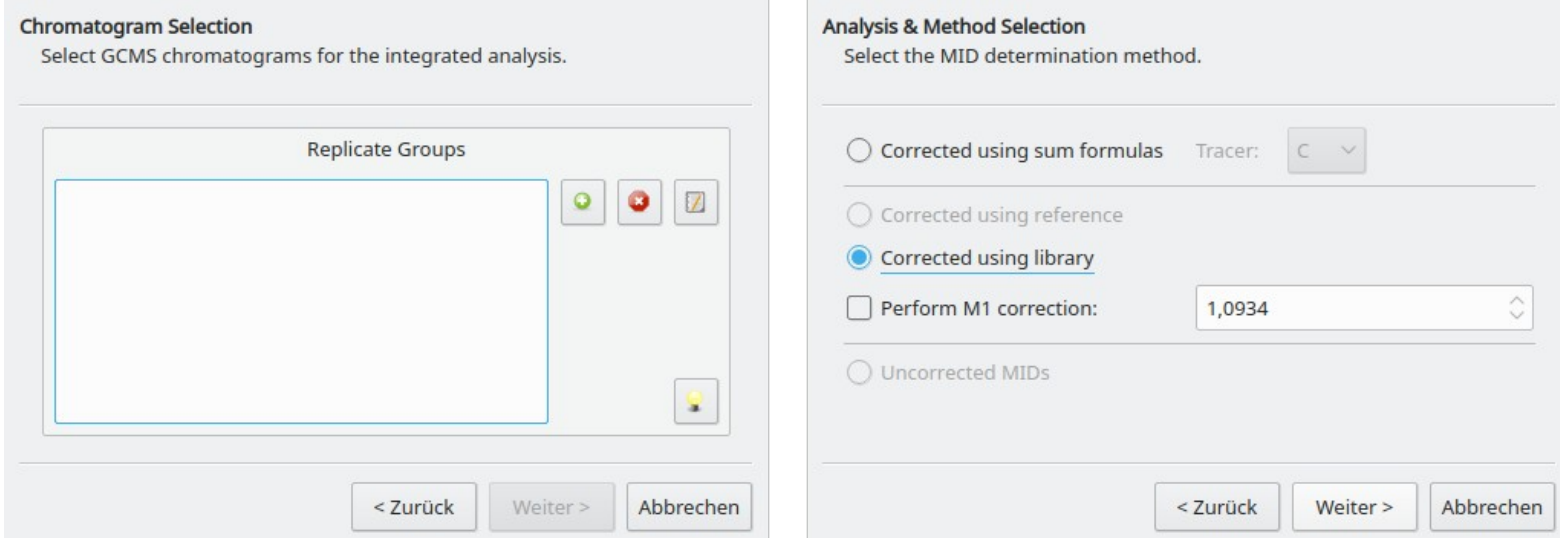

Figure 6: The MID wizard. The files for replicates (left) can either be select manually, using the plus button, or alternatively using the lightbulb button (see Note 3) for regular expression based replicate selection. In the analysis and methods section (right) it is important to select 'Corrected using library' to correct for natural isotopes abundance using unlabeled reference spectra. 


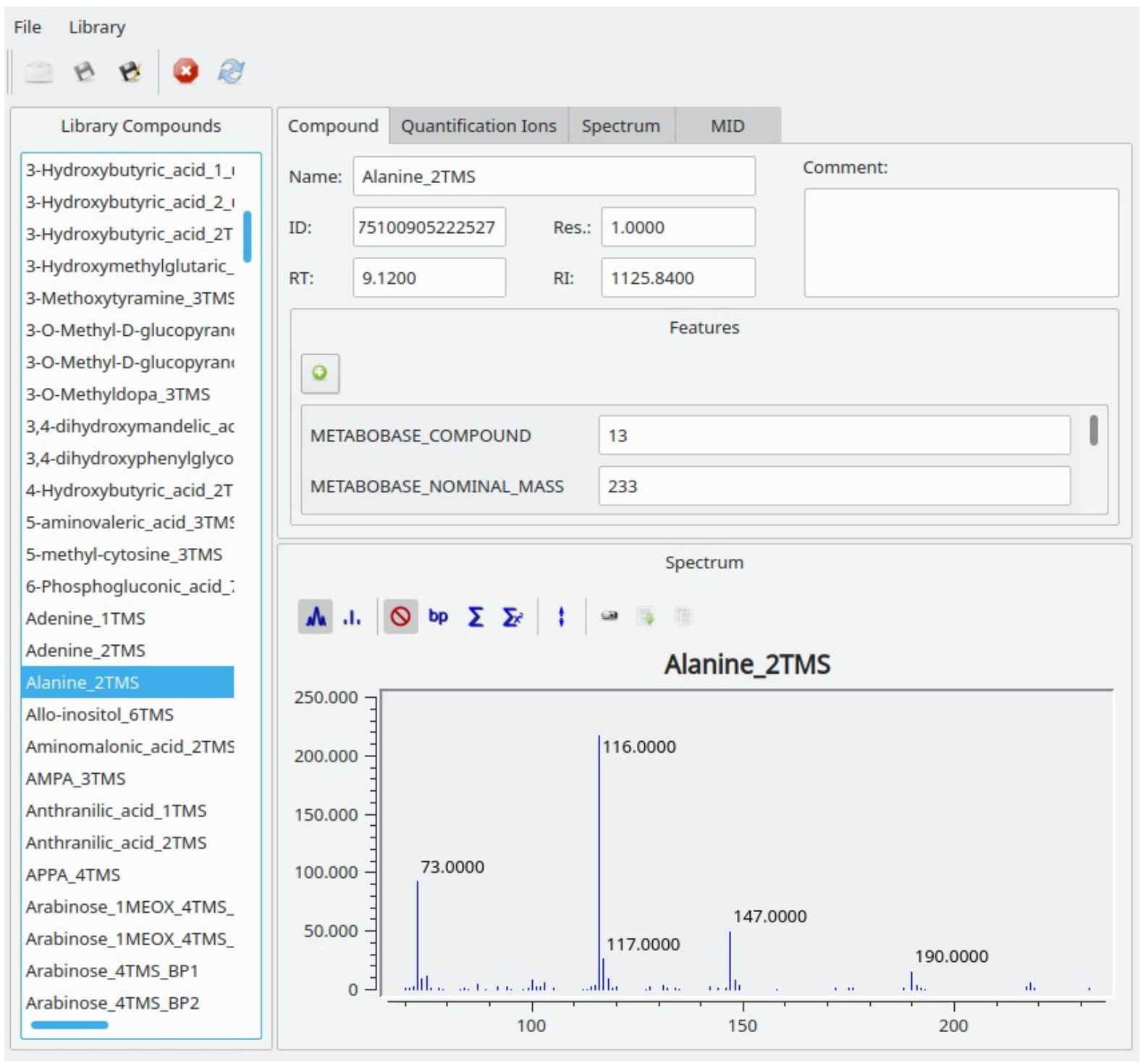

Figure 7: The library editor window of MetaboliteDetector. All library compounds are listed on the left. On the right, the stored data, including name, retention time, retention index, ions for quantification and MID determination and the compound spectrum, - can be reviewed and revised. 


\section{Spectra}

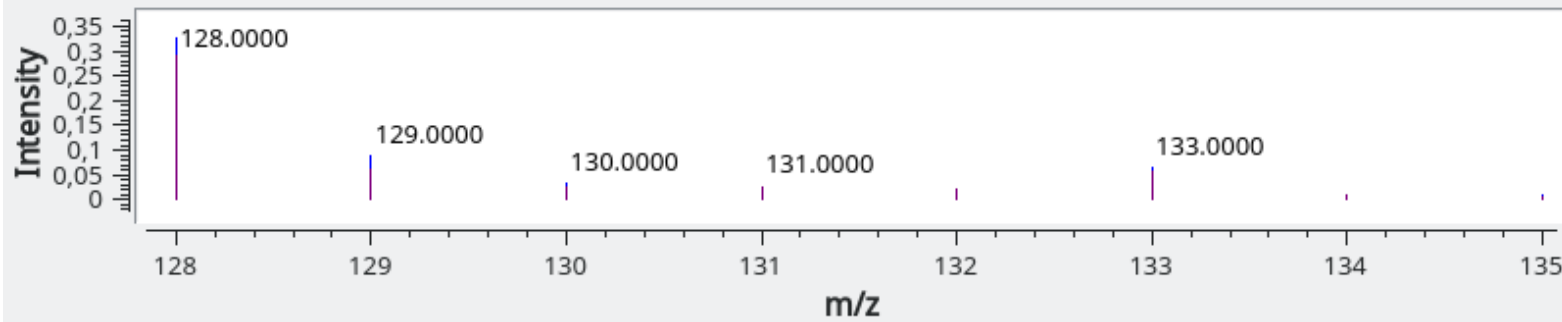

Spectra

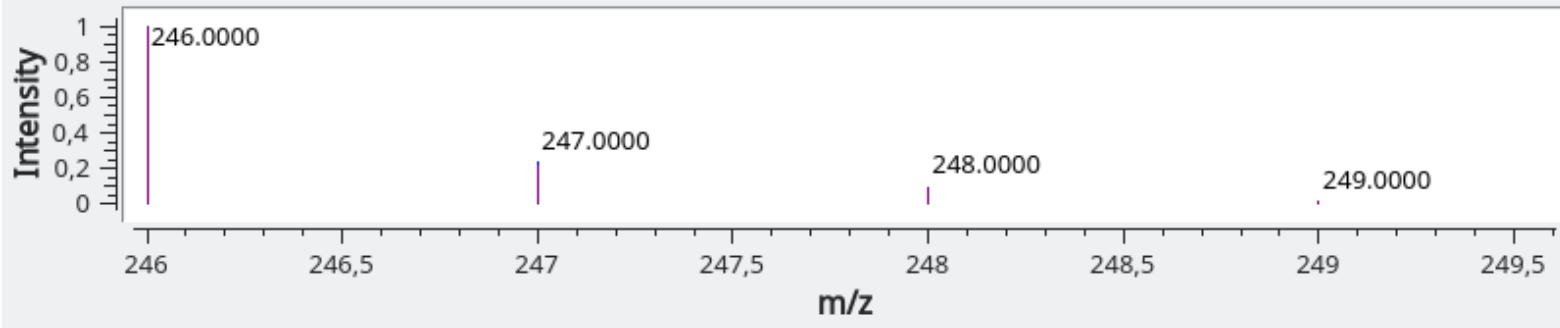

Figure 8: Spectrum of two fragments with mass 128 (upper spectrum) and 246 (lower spectrum) of an unlabeled metabolite. The upper spectrum has an unexpected high peak at M+5 (133) isotopomer, which suggests an artifact from insufficient deconvolution. In contrast, the lower spectrum has decreasing intensities for the mass isotopomers, as expected from an unlabeled sample. 


\section{Tables}

Table 1: Deconvolution settings for different GCMS instruments.

Instrument

Peak threshold Min. peak height

$2-10$

$5-20$

$5-20$

$20-50$
Bin/Scan

10

10

10

1
Deconvolution

width

7.0-8.0

1.0

3.0

40.0 


\section{References}

[1] C. Birkemeyer, A. Luedemann, C. Wagner, A. Erban, and J. Kopka, “Metabolome analysis: the potential of in vivo labeling with stable isotopes for metabolite profiling," Trends Biotechnol., vol. 23, no. 1, pp. 28-33, Jan. 2005.

[2] K. Zaitsu, Y. Hayashi, M. Kusano, H. Tsuchihashi, and A. Ishii, “Application of metabolomics to toxicology of drugs of abuse: A mini review of metabolomics approach to acute and chronic toxicity studies,” Drug Metab. Pharmacokinet., vol. 31, no. 1, pp. 21-26, Feb. 2016.

[3] M. I. Klapa and G. Stephanopoulos, "Metabolic Flux Analysis,” in Bioreaction Engineering, Berlin, Heidelberg: Springer Berlin Heidelberg, 2000, pp. 106-124.

[4] D. J. Creek, A. Chokkathukalam, A. Jankevics, K. E. V. Burgess, R. Breitling, and M. P. Barrett, "Stable Isotope-Assisted Metabolomics for Network-Wide Metabolic Pathway Elucidation,” Anal. Chem., vol. 84, no. 20, pp. 8442-8447, Oct. 2012.

[5] K. Hiller, C. Metallo, and G. Stephanopoulos, "Elucidation of Cellular Metabolism Via Metabolomics and Stable-Isotope Assisted Metabolomics,” Curr. Pharm. Biotechnol., vol. 12, no. 7, pp. 1075-1086, Jul. 2011.

[6] K. Hiller, C. M. Metallo, J. K. Kelleher, and G. Stephanopoulos, "Nontargeted Elucidation of Metabolic Pathways Using Stable-Isotope Tracers and Mass Spectrometry,” Anal. Chem., vol. 82, no. 15, pp. 6621-6628, Aug. 2010.

[7] D. Weindl, A. Wegner, and K. Hiller, "MIA: Non-targeted mass isotopolome analysis.,” Bioinformatics, p. btw317, Jun. 2016.

[8] K. Hiller et al., "NTFD--a stand-alone application for the non-targeted detection of stable isotope-labeled compounds in GC/MS data,” Bioinformatics, vol. 29, no. 9, pp. 1226-1228, May 2013.

[9] D. Weindl et al., "Bridging the gap between non-targeted stable isotope labeling and metabolic flux analysis,” Cancer Metab., vol. 4, no. 1, p. 10, Dec. 2016.

[10] X. Huang, Y.-J. Chen, K. Cho, I. Nikolskiy, P. A. Crawford, and G. J. Patti, "X ${ }^{13}$ CMS: Global Tracking of Isotopic Labels in Untargeted Metabolomics,” Anal. Chem., vol. 86, no. 3, pp. 1632-1639, Feb. 2014.

[11] C. Bueschl et al., "MetExtract II: A Software Suite for Stable Isotope-Assisted Untargeted Metabolomics,” Anal. Chem., vol. 89, no. 17, pp. 9518-9526, Sep. 2017.

[12] I. and Mark E. Jennings and D. E. Matthews*, "Determination of Complex Isotopomer 
Patterns in Isotopically Labeled Compounds by Mass Spectrometry,” 2005.

[13] K. Hiller, J. Hangebrauk, C. J??ger, J. Spura, K. Schreiber, and D. Schomburg, "Metabolite detector: Comprehensive analysis tool for targeted and nontargeted GC/MS based metabolome analysis,” Anal. Chem., vol. 81, no. 9, pp. 3429-3439, May 2009.

[14] J. Hummel, N. Strehmel, J. Selbig, D. Walther, and J. Kopka, "Decision tree supported substructure prediction of metabolites from GC-MS profiles,” Metabolomics, vol. 6, no. 2, pp. 322-333, Jun. 2010.

[15] S. Dietmair, N. E. Timmins, P. P. Gray, L. K. Nielsen, and J. O. Krömer, “Towards quantitative metabolomics of mammalian cells: Development of a metabolite extraction protocol,” Anal. Biochem., vol. 404, no. 2, pp. 155-164, Sep. 2010.

[16] B. Gonzalez, J. François, and M. Renaud, "A rapid and reliable method for metabolite extraction in yeast using boiling buffered ethanol,” Yeast, vol. 13, no. 14, pp. 13471355, Nov. 1997.

[17] S. Naz, D. C. Moreira dos Santos, A. García, and C. Barbas, “Analytical protocols based on LC-MS, GC-MS and CE-MS for nontargeted metabolomics of biological tissues,” Bioanalysis, vol. 6, no. 12, pp. 1657-1677, Jun. 2014.

[18] J. Lisec, N. Schauer, J. Kopka, L. Willmitzer, and A. R. Fernie, “Gas chromatography mass spectrometry-based metabolite profiling in plants,” Nat. Protoc., vol. 1, no. 1, pp. 387-396, Jun. 2006.

[19] J. Meiser, D. Weindl, and K. Hiller, “Complexity of dopamine metabolism,” Cell Commun. Signal., vol. 11, no. 1, p. 34, May 2013.

[20] J. Meiser et al., "Pro-inflammatory Macrophages Sustain Pyruvate Oxidation through Pyruvate Dehydrogenase for the Synthesis of Itaconate and to Enable Cytokine Expression.," J. Biol. Chem., vol. 291, no. 8, pp. 3932-46, Feb. 2016. 